\title{
Meningitis postquirúrgica. Características diferenciales de la meningitis aséptica post-quirúrgica
}

\author{
A. Ramos-Martínez; T. de las Heras-Carballo; C. Fernández-Mateos*; L. de Reina*; T. Álvarez de Espejo-Montiel; \\ N. Escamilla-Fernández; I. Sánchez-Romero** e I. Millán***
}

Servicios de Medicina Interna (Unidad de Infecciosas) Neurocirugía* y Microbiología**. Sección de Bioestadística***. Hospital Universitario Puerta de Hierro. Madrid.

Resumen

Introducción. La meningitis postquirúrgica es una complicación infrecuente que se acompaña de un incremento de la estancia hospitalaria y de una elevada mortalidad. Algunos de estos casos no son debidos a una verdadera infección sino a una inflamación aséptica de las meninges denominada meningitis aséptica post-quirúrgica (MAPQ). La adecuada identificación de estos casos permitiría una mejor utilización de los fármacos antimicrobianos.

Material y métodos. Estudio retrospectivo de los pacientes con meningitis postquirúrgica en un hospital terciario durante 14 años comparando las características clínicas de los pacientes con meningitis bacteriana postquirúrgica (MBP) frente a las de pacientes con MAPQ

Resultados. Durante el período analizado se identificaron 35 pacientes $(\mathbf{7 1} \%)$ con MBP y 14 pacientes $(29 \%)$ con MAPQ. La edad media de los pacientes con MBP fue similar a la de los pacientes con MAPQ. Hubo predominio de varones en el grupo de $\mathrm{MBP}(\mathbf{7 1 \%})$ en relación con los pacientes con MAPQ $(36 \%, p=0,020)$. La proporción de pacientes con hemorragia intracraneal tendió a ser más frecuente en pacientes que posteriormente desarrollaron MAPQ (9 pacientes, $64 \%$ ) que en los pacientes con MBP (12 casos, $34 \%$, $p=0,055)$. Los pacientes sometidos a craneotomía posterior $(\mathrm{p}=\mathbf{0 , 0 9 2})$ $y$ los que recibían tratamiento esteroideo $(p=0,051)$ mostraron una mayor tendencia a padecer MAPQ. Siete pacientes MBP (20\%) presentaron un recuento celular superior a 5000 células $/ \mathrm{mm}^{3}$ en el LCR, cifra no encontrada en ningún caso de MAPQ. No se detectaron diferencias en la glucorraquia y proteinorraquia entre ambos grupos. La bacterias más frecuentemente aisladas fueron Staphylococcus coagulasa negativa y $S$. aureus. En 5 pacientes (14\%) se aislaron bacilos gramnegativos no fermentadores (Pseudomonas aeruginosa y Acinetobacter spp). No hubo ningún fallecimiento atri-

Recibido: 4-01-08. Aceptado: 10-10-08 buido a meningitis postquirúrgica.

Conclusión. Los pacientes sometidos a neurocirugía que fueron ingresados por hemorragia cerebral, intervenidos de la fosa posterior o tratados con esteroides presentan una mayor tendencia a presentar MAPQ. Los recuentos celulares en LCR elevados (superiores a 5000 células $/ \mathbf{m m}^{3}$ ) sugieren la existencia de MBP.

PALABRAS CLAVE: Neurocirugía. Meningitis aséptica. Hemorragia intracraneal. Staphylococcus.

Postsurgical meningitis. Differential characteristics of aseptic postsurgical meningitis

\section{Summary}

Background. Postsurgical meningitis is a rare complication that is accompanied by an increase of hospital stay and high mortality. Some of these cases are not due to a true infection but due to an aseptic inflammation of the meninges denominated aseptic postsurgical meningitis (APSM). Proper identification of these cases would allow better use of antimicrobial drugs.

Methods. A retrospective study of patients with postsurgical meningitis in a universitary hospital for 14 years. We describe the clinical characteristics of patients with postsurgical bacterial meningitis (PBM) compared to those of patients with APSM.

Results. During the studied period 35 patients (71\%) with PBM and 14 patients (29\%) with (APSM) were identified. The mean age of patients with PBM was similar to that of patients with APSM. There was a male predominance in the group of PBM (71\%) compared with patients with APSM $(36 \%, p=0.020)$. Patients with intracranial hemorrhage tended to present more cases of APSM (64\%) than of PBM (34\%, $p=0.055)$. Patients undergoing posterior fossa craniotomy $(p=$

Abreviaturas. LCR: líquido cefalorraquideo. MAPQ: meningitis aséptica post-quirúrgica. MP: meningitis postquirúrgica. $M B P$ : meningitis postquirúrgica bacteriana. 
$0.092)$ and those receiving steroids $(p=0.051)$ showed a greater tendency to suffer APSM. It was also noted a trend towards present $\mathrm{PBM}$ in patients who had suffered an infection in the previous month $(p=0.072)$. There were seven patients with PBM (20\%) with a cell count above 5000 cells $/ \mathrm{mm}^{3}$ in CSF, values not found in any patients with APSM. No differences were detected in the glycorrachia and proteinorrachia between the two groups. The most common bacteria isolated were coagulase negative Staphylococcus and $S$. aureus. In 5 patients (14\%) non fermenting gram-negative bacillus (Pseudomonas aeruginosa and Acinetobacter spp) were isolated. There were no deaths attributed to any type of postsurgical meningitis.

Conclusion. Patients admitted for brain haemorrhage, undergoing posterior fossa surgery or receiving steroids tend to develop APSM. A CSF cell count above 5000 cells $/ \mathbf{~ m m}^{3}$ strongly suggests $\mathrm{MBP}$.

KEY WORDS: Neurosurgery. Meningitis aseptic. Intracranial hemorrhages. Staphylococcus.

\section{Introducción}

La meningitis postquirúrgica (MP) es una complicación infrecuente $(0,3-1,5 \%)$ pero de indudable transcendencia clínica $^{12,16,18}$. Además de ser responsable del empleo de recursos económicos y del incremento de las estancias hospitalarias, puede ocasionar el fallecimiento del paciente $(20-50 \%)^{6,7}$.

La presentación clínica de estos enfermos suele consistir en fiebre, meningismo y/o alteraciones del nivel de conciencia ${ }^{15}$. Los fenómenos inflamatorios originados por determinados tipos de tumores (especialmente si tienen componente quístico), la manipulación tisular durante la cirugía o la reabsorción sanguínea y de restos óseos pueden ser responsables de un cuadro clínico similar ${ }^{2,416}$. También son semejantes las alteraciones del líquido cefalorraquídeo (LCR) que son debidas a inflamación sin infección ${ }^{1}$. Diferenciar estos casos, definitorios de una variedad de meningitis denominada meningitis aséptica post-quirúrgica (MAPQ), de los pacientes con meningitis bacteriana postquirúrgica (MBP) constituye una complicada tarea que con frecuencia no consigue evitar la administración de terapia antimicrobiana innecesaria en algunos aspectos ${ }^{3,4,11}$.

El objetivo del trabajo fue describir las características clínicas de las meningitis postquirúrgicas en nuestro medio y conocer las posibles diferencias entre las MAPQ y las MBP.

\section{Material y métodos}

Se realizó un estudio observacional de una cohorte de pacientes mayores de 18 años que desarrollaron meningitis postquirúrgica durante su estancia hospitalaria. El centro donde se realizó el estudio es un hospital universitario con 500 camas y unos 15.000 ingresos anuales y en el que radica un servicio de neurocirugía de referencia para otras áreas geográficas.

Se incluyeron todos aquellos pacientes con meningitis adquirida en el hospital desde Enero de 1994 hasta Diciembre de 2007. Esta entidad se definió por la presencia de fiebre $\left(>38^{\circ} \mathrm{C}\right)$, cefalea, rigidez de nuca, signos meníngeos, disminución del nivel de conciencia, pleocitosis (más de 5 células $/ \mathrm{mm}^{3}$ en el LCR), y al menos una de las siguientes alteraciones del LCR: hipoglucorraquia (menor $40 \mathrm{mg} / \mathrm{dl} \mathrm{o}$ menor del 50\% de la glucemia) o hiperproteinorraquia (valor superior a $60 \mathrm{mg} / \mathrm{dl})^{8}$. En los pacientes que tenían una punción del LCR con aspecto hemático una relación leucocitos/ hematíes $>100$ era requerida para su inclusión. Cuando la tinción de Gram o el cultivo objetivaron microorganismos se consideró la existencia de MBP y cuando los resultados microbiológicos eran negativos y el paciente no había recibido antibiótico durante la semana previa, meningitis aséptica post-quirúrgica. En estos pacientes el tratamiento antimicrobiano pautado de modo empírico era retirado tras conocer la negatividad del cultivo. Si el paciente había recibido antibióticos durante la semana previa y el cultivo era negativo el caso era considerado como meningitis de etiología indeterminada y por tanto no era incluido en el estudio. En los pacientes que presentaran más de un episodio sólo se consideraría el primero de ellos.

De cada uno de los pacientes se consideró su edad, sexo, antecedentes patológicos y tratamientos previos, tiempo transcurrido desde la cirugía hasta la aparición de meningitis, tipo de intervención quirúrgica, derivación de LCR, existencia de fístula de LCR, presentación clínica, analítica de LCR (glucosa, células, fórmula y proteínas), tinción de Gram y cultivo de LCR, hemocultivos, tratamiento empleado, días de estancia hospitalaria y posible fallecimiento.

Las variables categóricas se expresaron en porcentajes. Las variables continuas se expresaron con su media y desviación estándar. La hipótesis de normalidad fue evaluada mediante los test de Kolmogorov-Smirnov y de ShapiroWilk. Para evaluar las diferencias de las medias en el análisis univariante se emplearon la $t$ de Student o el test no paramétrico de Mann-Whitney, si la normalidad no pudo ser asumida. Para comparar las variables categóricas se empleó la prueba exacta de Fisher si la muestra era menor a 5 , y si no la prueba de la Chi cuadrado. Se consideró significación estadística un valor de p a una cola menor de 0,05 .

\section{Resultados}

Durante el período de estudio se identificaron cincuenta y cinco pacientes que cumplían criterios de MP. Se desestimaron seis casos que tenían un cultivo de LCR negativo y 
Tabla 1

Factores predisponentes de meningitis postquirúrgica

\begin{tabular}{|l|c|c|c|c|}
\hline & $\begin{array}{c}\text { Meningitis bacteriana } \\
\text { postquirúrgica (n=35) }\end{array}$ & $\begin{array}{c}\text { Meningitis aséptica } \\
\text { post-quirúrgica (n=14) }\end{array}$ & p & RR (IC 95\%) \\
\hline Diabetes mellitus & $2(6)$ & $1(7)$ & 0,850 & $0,80(0,1-8,1)$ \\
\hline Inmunodepresión $^{1}$ & $4(11)$ & $1(7)$ & 0,654 & $1,6(0,2-13,1)$ \\
\hline Corticoides $^{2}$ & $9(26)$ & $8(57)$ & 0,051 & $0,45(0,2-0,9)$ \\
\hline Meningitis postquirúrgica previa $^{\prime}$ & $4(11)$ & $1(7)$ & 0,654 & $1,6(0,2-13,1)$ \\
\hline Fístula de LCR & $3(6)$ & $0(0)$ & 0,258 & \\
\hline Portador de DVP $^{4}$ & $8(23)$ & $4(29)$ & 0,674 & $0,8(0,3-2,2)$ \\
\hline DVE $^{5}$ & $11(31)$ & $3(21)$ & 0,483 & $1,5(0,5-4,5)$ \\
\hline Craneotomía fosa posterior $^{3}$ & $5(14)$ & $5(36)$ & 0,092 & $0,40(0,1-1,2)$ \\
\hline Craneotomía otras localizaciones $^{5}$ & $27(77)$ & $8(57)$ & 0,161 & $1,3(0,8-2,2)$ \\
\hline Otras intervenciones $^{6}$ & $3(9)$ & $1(7)$ & 0,868 & $1,2(0,1-10,6)$ \\
\hline
\end{tabular}

1 Pacientes en tratamiento con fármacos imunosupresores o quimioterapia, 2 Dosis equivalente de dexametasona superiores a $8 \mathrm{mg} /$ día, 3 Presencia de fístula de LCR previa a la meningitis, 4 DVP: derivación ventrículoperitoneal previa a la meningitis, 5 Craneotomia fosa anterior (15 pacientes), de fosa media (5 pacientes), craneotomía transesfenoidal ( 7 pacientes), 6 Intervenciones de la columna vertebral. Los porcentajes se expresan entre paréntesis. RR: riesgo relativo, IC 95\%: índice de confianza al 95\%. Los porcentajes se expresan entre paréntesis

que habían recibido antibióticos durante los siete días previos por considerar que se trataban de meningitis de causa indeterminada. De los cuarenta y nueve pacientes restantes, treinta y cinco (71\%) tuvieron un cultivo de LCR positivo (MBP) y catorce (29\%) presentaron una tinción de Gram y un cultivo negativo constituyendo el grupo de pacientes con meningitis aséptica post-quirúrgica.

La edad media de los pacientes con MBP era 51,6 \pm 20 años (rango 18-83 años) y era similar a la de los pacientes con MAPQ, 50,6 \pm 18 años (rango 25-81 años, $\mathrm{p}=0,868$ ). La proporción de varones entre los casos de MBP (25 pacientes, 71\%) fue superior a la de MAPQ (5 pacientes, $36 \%, p=0,02$ ). No se detectaron diferencias en el número de pacientes diabéticos o inmunodeprimidos entre ambos grupos.

Las patologías que con más frecuencia determinaron la intervención quirúrgica fueron hemorragia intracraneal (21 pacientes, $43 \%$ ) y tumor encefálico (20 casos, $41 \%$ ). El resto de enfermos padecían aneurisma intracraneal (2 casos), hernia discal (2 casos), estenosis canal cervical
( 2 casos), síndrome de silla turca vacía ( 1 caso) y malformación Arnold-Chiari II (1 caso). La proporción de pacientes con hemorragia intracraneal fue más elevada en pacientes que posteriormente desarrollaron MAPQ (9 pacientes, 64\%) que en los pacientes con MBP (12 casos, $34 \%, \mathrm{p}=0,055, \mathrm{RR}=0,53$, RR $95 \%=0,3-0,9)$. Los pacientes sometidos a craneotomía posterior $(\mathrm{p}=0,092)$ y los que recibían tratamiento esteroideo $(\mathrm{p}=0,051)$ mostraron una mayor tendencia a padecer MAPQ. También se observó una tendencia hacía padecer MBP en pacientes que habían padecido una infección en el mes previo $(\mathrm{p}=0,072$, Tabla 1).

El período de tiempo transcurrido entre la intervención quirúrgica y la aparición de los síntomas fue similar en ambos grupos. La mediana en los casos de MAPQ fue de 9 días y de 10 días en los que desarrollaron $\mathrm{MBP}(\mathrm{p}=0,874)$. Las manifestaciones clínicas que presentaban los pacientes se describen en la tabla 2. Se apreció fiebre en el $80 \%$, cefalea en el $23 \%$ de los pacientes y disminución del nivel de conciencia en $34 \%$ de los enfermos. Solo en 2 pacientes 
Tabla 2

Manifestaciones clínicas de pacientes con meningitis postquirúrgica

\begin{tabular}{|l|c|c|c|c|}
\hline & $\begin{array}{c}\text { Meningitis bacteriana } \\
\text { postquirúrgica (n=35) }\end{array}$ & $\begin{array}{c}\text { Meningitis aséptica } \\
\text { post-quirúrgica (n=14) }\end{array}$ & $\mathrm{p}$ & RR (RR al 95\%) \\
\hline Fiebre & $28(80)$ & $12(86)$ & 0,640 & $0,9(0,7-1,2)$ \\
\hline Cefalea & $8(23)$ & $6(43)$ & 0,160 & $0,53(0,1-1,2)$ \\
\hline Disminución conciencia & $12(34)$ & $1(7)$ & 0,075 & $4,8(0,7-33,5)$ \\
\hline
\end{tabular}

Los porcentajes se expresan entre paréntesis

Tabla 3

Etiología de 35 casos de meningitis postquirúrgica bacteriana

\begin{tabular}{|l|c|c|}
\hline & Meningitis bacteriana postquirúrgica (n=35) & Porcentaje \\
\hline Staphylococcus aureus & 6 & 17 \\
\hline Staphylococcus coagulasa negativo & 15 & 43 \\
\hline Streptococcus spp & 2 & 3 \\
\hline Streptococcus pneumoniae & 1 & 6 \\
\hline Enterococcus spp & 2 & 3 \\
\hline Listeria monocitogenes & 1 & 6 \\
\hline Klebsiella pneumoniae & 2 & 6 \\
\hline Pseudomonas aeruginosa & 3 & 9 \\
\hline Acinetobacter spp & 1 & 3 \\
\hline Escherichia coli & 2 & 6 \\
\hline
\end{tabular}

$(3,7 \%)$ tenían los tres síntomas de modo simultáneo. Los pacientes con MBP, presentaron disminución del nivel de conciencia con mayor frecuencia que los pacientes con MAPQ ( $p=0,075$, tabla 2).

La cifra media de células en los pacientes con MBP fue de $8607 \pm 30602$, mediana 420, rango 41-160000 células y la de pacientes con MAPQ: media $785 \pm 761$, mediana 520, rango 49-2800 ( $\mathrm{p}=0,647)$. Siete pacientes con MBP $(20 \%)$ tuvieron un recuento celular en LCR superior a 5000 células $/ \mathrm{mm}^{3}$. Estos valores no fueron encontrados en ningún caso de MAPQ. Hubo un predominio de leucocitos polimorfonucleares en el 78\% de los pacientes con MAPQ y en el 79\% de los pacientes con MBP. La glucorraquia en pacientes con MBP (51 \pm 20 , mediana 48, rango 10-225 $\mathrm{mg} / \mathrm{dl})$ también fue similar a la de pacientes con MAPQ (49 \pm 38 , mediana 40, rango $10-163 \mathrm{mg} / \mathrm{dl}, \mathrm{p}=0,689)$. La proteinorraquia tampoco arrojó valores que permitieran diferenciar ambos grupos $(233 \pm 271$, mediana 155 , rango $10-1430 \mathrm{mg} / \mathrm{dl}$ en pacientes con MBP y $169 \pm 117$, mediana 157 , rango $15-440 \mathrm{mg} / \mathrm{dl} ; \mathrm{p}=0.729$ ).

La etiología de los casos de MBP figuran en la tabla 3. Los estafilococos coagulasa negativa y Staphylococcus aureus fueron las bacterias más frecuentemente aisladas. En 6 pacientes se aisló el mismo germen en el LCR y en los 
hemocultivos: Staphylococcus spp. coagulasa negativa (4 pacientes), E. coli y Klebsiella pneumoniae (un paciente, respectivamente).

\section{Discusión}

Entre las dificultades diagnósticas de los pacientes con MP destaca la diferenciación de MBP de las MAPQ $\mathrm{o}$ asépticas ${ }^{15,11}$. Aunque en nuestra serie las MAPQ constituyeron algo menos del tercio de los pacientes, en otras series fue el grupo mayoritario ${ }^{2,15}$, lo que permite valorar la dimensión de este problema. La correcta identificación de los casos de MAPQ permitiría disminuir el número de pacientes que reciben antibioterapia y con ello acortar las estancias hospitalarias y disminuir los gastos de farmacia, efectos adversos y la presión antibiótica sobre las bacterias hospitalarias ${ }^{13}$.

La proporción pacientes diabéticos o bajo tratamiento inmunosupresor fue similar en ambos grupos. No fue así el porcentaje de pacientes que recibían corticoides, mayor en pacientes con MAPQ, probablemente por tratarse de pacientes con hipertensión intracraneal. También se debe destacar que los 3 casos con fístula de LCR de la serie padecieron $\mathrm{MBP}^{15}$ lo que resulta lógico por la existencia de una solución de continuidad de las cubiertas encefálicas ${ }^{17}$. Ha sido comunicado con anterioridad que los pacientes intervenidos de la fosa posterior presentan un mayor riesgo de $\mathrm{MAPQ}^{4,15}$, especialmente los que desarrollan meningocele ${ }^{10}$. También en nuestros pacientes se observó una mayor proporción de pacientes con hemorragias intracraneales entre los pacientes que luego desarrollaron MAPQ, esta tendencia, sin embargo, no ha sido evidenciado en otras series ${ }^{2}$.

Los síntomas suelen aparecer durante las tres primeras semanas después de la cirugía ${ }^{2}$. En nuestros pacientes no hubo diferencias en el momento de aparición de la sintomatología entre ambos grupos. Este hallazgo contrasta con lo publicado en la literatura referente a una aparición más temprana de la fiebre en los pacientes con MAPQ. Esta diferente presentación se ha relacionado con la inmediatez de los fenómenos inflamatorios que se desencadenan durante la intervención quirúrgica en contraposición con el tiempo de incubación que toda infección requiere para alcanzar una masa de microorganismos capaz de originar determinados síntomas y signos ${ }^{4}$. Debido al número relativamente escaso de investigaciones realizadas sobre este tema, deberemos aguardar a la publicación de series de MP más amplias para comprobar si detectan o no diferencias en el tiempo transcurrido desde la cirugía entre las MPB y las MAPQ.

La disminución del nivel de conciencia en pacientes neuroquirúrgicos puede deberse a su enfermedad de base o al empleo de sedantes. Sin embargo, este signo también se ha relacionado con las $\mathrm{MBP}^{4}$. Este ominoso signo apareció con más frecuencia en pacientes con MBP, aunque no alcanzó significación estadística. Por tanto, en enfermos sometidos a una intervención neuroquirúrgica, la aparición de fiebre y disminución del nivel de conciencia debería alertar sobre la posibilidad de MBP, sobre todo si tienen una derivación ventricular externa o una fístula de $\mathrm{LCR}^{4}$.

Debe destacarse la elevada proporción de Staphylococcus spp. coagulasa negativa que hemos encontrado en nuestra serie, lo que coincide con otras series'. Se debe destacar que el $15 \%$ de los casos de MBP estaban causados por bacterias gramnegativas no fermentadoras (Pseudomonas spp. y Acinetobacter spp.) que suelen presentar un perfil de resistencia complejo y que plantean un problema serio respecto a su cobertura antibiótica empírica ${ }^{6}$.

Ningún síntoma o signo aislado posee la capacidad de descartar o confirmar la existencia de MBP. No obstante, en nuestra serie se ha observado que una pleocitosis superior a 5000 leucocitos $/ \mathrm{mm}^{3}$ sugiere claramente un origen bacteriano de la meningitis ${ }^{4,5}$. Sin embargo, y a pesar de lo publicado con anterioridad, no se ha hallado un nivel de hipoglucorraquia que sólo se haya objetivado en pacientes con $\mathrm{MBP}^{4}$. Tampoco se ha observado que la pleocitosis mononuclear sea más frecuente en los pacientes con $\mathrm{MAPQ}^{2,4}$, por lo que el predominio de leucocitos polimorfonucleares no resulta útil para diferenciar MAPQ de $\mathrm{MBP}^{3}$. Entre los marcadores bioquímicos del LCR estudiados para diferenciar estas dos entidades destaca la interleuquina-1 que presenta un excelente valor predictivo negativo y no parece modificarse por el tratamiento corticoideo $^{13}$. Desafortunadamente, la determinación de esta citoquina es una técnica experimental no disponible en la mayoría de las instituciones. Considerando las limitaciones del análisis convencional de LCR y la gravedad de la MBP, resulta razonable en muchos casos comenzar un tratamiento antibiótico empírico hasta disponer de los resultados de microbiología.

Este trabajo presenta una serie de limitaciones que condicionan una cierta precaución al intentar extrapolar sus resultados. Entre ellas destaca ser un estudio retrospectivo lo que implica que la información accesible haya podido ser incompleta en algunos casos. Así mismo, el escaso tamaño muestral ha podido impedir demostrar diferencias estadísticamente significativas en algunas variables. También se debe señalar que no se hizo un análisis multivariante por las escasas diferencias mostradas en el análisis univariante.

Considerando la situación actual resulta evidente que son necesarias nuevas investigaciones que permitan avanzar en el conocimiento y manejo práctico de los pacientes neuroquirúrgicos con meningitis aséptica. Esto permitiría acortar el ingreso de algunos pacientes y evitar la toxicidad, coste e incremento de resistencias bacterianas asociados al 
uso no discriminativo de antibióticos.

\section{Bibliografía}

1. Blomstedt, G.C.: Infections in neurosurgery: A restrospective study of 1143 patients and 1517 operations. Acta Neurochir (Wien) 1985; 78: 81-90.

2. Carmel, P.W., Fraser, R.A.R., Stein, B.M.: Aseptic meningitis following posterior fossa surgery in children. J Neurosurgery 1974; 41: 44-48.

3. Filete, V., Parés, P., Molet, J., Oliver, B., Tresserras, P., Bartumeus, F.: Estimación del coste económico atribuible a la infección postoperatoria en neurocirugía. Neurocirugía 1998; 9: 108-114.

4. Forgacs, P., Geyer, C.A., Freidberg, S.R.: Characterization of aseptic postsurgical meningitis after neurological surgery. Clin Infect Dis 2001; 32: 179-185.

5. Gabriela, B., Sanchez, G.B., Kaylie, D.M., et al: Aseptic postsurgical meningitis following cerebellopontine angle tumor surgery. Otolaryngol Head Neck Surg 2008; 128: 368373.

6. Ho, Y.H., Wang, L.S., Chao, H.J., Chang, K.C., Su, C.F.: Successful treatment of meningitis caused by multidrug-resistant Acinetobacter baumannii with intravenous and intrathecal colistin. J Microbiol Immunol Infect 2007; 40: 537-544.

7. Hollman, H.D., Haines, S.J.: Risk factors for postoperative neurosurgical wound infection: a case-control study. J Neurosurgery 1986; 64: 902-906.

8. Horan, T.C., Gaynes, R.P., Martone, W.J., Jarvis, W.R., Emori, T.G.: CDC definitions of nosocomial surgical site infections, 1992: A modification of CDC definitions of surgical wound infections. Infect Control Hosp Epidemiol 1992; 13: 606-608.

9. Huang, C.R., Lu, C.H., Wu, J.J., et al: Coagulase-negative staphylococcal meningitis in adults: clinical characteristics and therapeutic outcomes Infection 2005; 33: 56-60.

10. Jacobs, G.B., Klemme, W.M., Murphree, H.C.: Aseptic meningitis and pseudomeningocele formation as a complication of surgery in the posterior fossa: Pac Med Surg 1966; 6: 320-324.

11. Kaufman, B.A., Tunkel, A.R., Pryor, J.C., Dacey, R.G.

\section{Comentario al trabajo Meningitis postquirúrgica. Características diferenciales de la meningitis aséptica post-quirúrgica de A Ramos Martinez y cols.}

Globalmente, la incidencia de la infección del sistema nervioso central post-quirúrgica oscila entre el 5-7\% sin profilaxis quirúrgica a menos de un $1 \%$ en pacientes con profilaxis: $0,8 \%$ en pacientes con craniotomía y $0.4 \%$ en pacientes con cirugía de columna ${ }^{3}$.
Jr.: Meningitis in the neurosurgical patient. Infect Dis Clin North Am 1990; 4: 677-701.

12. Leib, S.L., Boscacci, R., Gratzl, O., Zimmerli, W.: Predictive value of cerebrospinal fluid (CSF) lactate level versus $\mathrm{CSF} / \mathrm{Blood}$ glucose ratio for the diagnosis of bacterial meningitis following neurosurgery. Clin Inf Dis 1999; 29: 69-74.

13. Lopez-Cortes, L.F., Marquez-Arbizu, R., JimenezJimenez, L.M., et al: Cerebrospinal fluid tumor necrosis factor- $\beta$, interleukin- $1 \beta$, interleukin- 6 , and interleukin- 8 as diagnostic markers of cerebrospinal fluid infection in neurosurgical patients. Crit Care Med 2000; 28: 215-219.

14. Palabiyikoglu, I., Tekeli, E., Cokca, F., et al.: Nosocomial meningitis in a university hospital between 1993 and 2002. J Hosp Infect 2006; 62: 94-97.

15. Ross, D., Rosegay, H., Pons, V.: Differentiation of aseptic and bacterial meningitis in postoperative neurosurgical patients. J Neurosurg 1988; 69: 669-674.

16. Salord, F., Boussaid, O., Eynard, N., Perret, C., Grando, J., Chacornac, R.: Interet du dosage du D(-) lactate pour le diagnostic rapide de meningite après craniotomie: étude preliminaire. Ann Fr Anesth Reanim 1994; 13: 647653.

17. Siegman-Igra, Y., Bar-Yosef, S., Gorea, A., Avram, J.: Nosocomial acinetobacter meningitis secondary to invasive procedures: report of 25 cases and review. Clin Infect Dis 1993; 17: 843-849.

18. Tavares, W.M., Machado, A.G., Matushita, H., Plese, J.P.: CSF markers for diagnosis of bacterial meningitis in neurosurgical postoperative patients. Arq Neuropsiquiatr 2006; 64: 592-595.

Ramos-Martínez, A.; de las Heras-Carballo, T.; FernándezMateos, C.; de Reina, L,; Álvarez de Espejo-Montiel, T.; Escamilla-Fernández, N.; Sánchez-Romero I.; Millán I.: Meningitis postquirúrgica. Características diferenciales de la meninigits aséptica post-quirúrgica. Neurocirugía 2009; 20: 103-109.

Correspondencia postal: Antonio Ramos Martínez. Medicina Interna III: Universidad Autónoma de Madrid. San Martín de Porres 4, Madrid 28034. 
meningitis postquirúrgicas son asépticas y ocurren con mayor frecuencia en niños y en cirugía de la fosa posterior. Las características citobioquímicas del LCR de estos pacientes son muy similares a las de los pacientes con una meningitis post-quirúrgica bacteriana. El único dato clínico que se ha postulado como diferencial es la aparición más precoz de la meningitis aséptica respecto a la meningitis postquirúrgica bacteriana, que se suele producir con una media de 10-14 días si bien hasta el 30\% de los pacientes que desarrollan meningitis postquirúrgica bacteriana la desarrollan en la primera semana de la cirugía ${ }^{2,4}$. Los resultados del estudio de Ramos y cols. certifican las dificultades en la diferenciación de ambas entidades por los datos clínicos y citobioquímicos del LCR por lo que el cultivo de LCR es la única prueba que permite distinguir ambos cuadros. Desde el punto de vista práctico, dado que existe evidencia de que en los pacientes con cultivo negativo de LCR en cirugía con alto riesgo de meningitis aséptica (cirugía de fosa posterior, importante sangrado) es seguro retirar los antibióticos cuando se confirme dicha negatividad ${ }^{4}$ creo que se debe generalizar esta práctica siempre que se compruebe que no existan circunstancias que favorezca un falso negativo del cultivo de LCR (p.ej antibioterapia previa).

\section{Bibliografía}

1. Carmel, P.W., Greif, L.K.: The aseptic meningitis syndrome: a complication of posterior fossa surgery. Pediatr Neurosurg 1993 Sep-Oct; 19: 276-280.

2. Korinek, A.M., Baugnon, T., Golmard, J.L., van Effenterre, R., Coriat, P., Puybasset, L.: Risk Factors for Adult Nosocomial Meningitis after Craniotomy: Role of Antibiotic Prophylaxis. Neurosurgery 2006; 59: 126-133.

3. McClelland, III S., Hall, W.A.: Postoperative Central Nervous System Infection: Incidence and Associated Factors in 2111 Neurosurgical Procedures. Clin Infect Dis 2007 July 1, 2007; 45: 55-59.

4. Zarrouk, V., Vassor, I., Bert, F., Bouccara, D., Kalamarides, M., Bendersky, N., et al.: Evaluation of the management of postoperative aseptic meningitis. Clin Infect Dis 2007 Jun 15; 44: 1555-1559. 